\title{
Comparative mutational analysis of distal colon cancer with rectal cancer
}

\author{
ZHIYONG ZHANG $^{1 *}$, AILI WANG ${ }^{2,3^{*}}$, XUAN TANG $^{2,4}$, YING CHEN $^{2,3}$, ERJIANG TANG ${ }^{2,3}$ and HUIHONG JIANG ${ }^{2,4}$ \\ ${ }^{1}$ Department of General Surgery, Zhuji People's Hospital of Zhejiang Province, Zhuji, Zhejiang 311800; \\ ${ }^{2}$ Institute of Gastrointestinal Surgery and Translational Medicine; ${ }^{3}$ Center for Clinical Research and \\ Translational Medicine, Yangpu Hospital; ${ }^{4}$ Department of General Surgery, Yangpu Hospital, \\ Tongji University School of Medicine, Shanghai 200090, P.R. China
}

Received February 25, 2019; Accepted December 12, 2019

DOI: $10.3892 / \mathrm{ol} .2020 .11269$

\begin{abstract}
Distal colon and rectal cancer are associated with each other but display distinct clinical behavior; however, the genetic basis for these differences is poorly understood. In the present study, a systematic comparison of mutational profiles between 137 distal colon and 125 rectal cancer samples was performed based on the data from the Memorial Sloan Kettering Cancer Center. Tumor mutational burden analysis showed that distal colon and rectal cancer harbored a similar burden of $\sim 5.9$ mutations/megabase, irrespective of the mismatch repair status. Comparison of significantly mutated genes between the groups determined that B-Raf proto-oncogene serine/threonine kinase mutations were enriched in distal colon cancer, whilst RAS and SMAD family member 4 (SMAD4) mutations were significantly more frequent in rectal cancer. Furthermore, two novel and potentially targetable hotspot mutations (APC regulator of WNT signaling pathway R876* and SMAD4 R361) were identified, which were enriched in rectal cancer compared with distal colon cancer. Overall, the results of the present study showed that the mutation profiles of distal colon and rectal cancer were largely similar, but distinct in specific key genetic events, which may provide valuable information for improving the management of patients with the disease.
\end{abstract}

Correspondence to: Dr Huihong Jiang, Department of General Surgery, Yangpu Hospital, Tongji University School of Medicine, 450 Tengyue Road, Shanghai 200090, P.R. China

E-mail: 1710470@tongji.edu.cn

Dr Erjiang Tang, Center for Clinical Research and Translational Medicine, Yangpu Hospital, Tongji University School of Medicine, 1015 Hejian Road, Shanghai 200090, P.R. China

E-mail: tangerjiang1988051@163.com

${ }^{*}$ Contributed equally

Key words: distal colon cancer, rectal cancer, mutational analysis, hotspot

\section{Introduction}

Colorectal cancer (CRC) is the third most prevalent malignancy, with an estimated 1.4 million new cases and 693,900 deaths worldwide in 2012 (1). Tumor sidedness has emerged as an important prognostic and predictive factor in the treatment of patients with CRC (2). Multiple studies have demonstrated that proximal colon cancer exhibits significantly different clinical and biological features compared with distal colon or rectal cancer (3). From a molecular point of view, the former is generally diploid and exhibits higher rates of microsatellite instability (MSI), whereas chromosomal instability (CIN) is more frequent in the latter (4). Anatomically, they have a different embryological origin, the proximal colon is derived from the midgut and the distal colon and rectum are derived from the hindgut (3). Therefore, traditionally, patients with distal colon and rectal cancer have frequently been grouped together in clinical or scientific research. However, there is increasing evidence that distal colon and rectal cancer are related to each other but are distinct in regard to their clinical behavior, including the patterns of metastasis, response to treatment and clinical outcome (5-7). However, to the best of our knowledge, the underlying biological carcinogenic backgrounds of the two types of cancer have not been investigated.

CRC is a highly complex and heterogeneous disease involving somatic mutation events associated with the interplay and crosstalk between critical oncogenic pathways $(8,9)$. Tie et al (10) reported that distal colon cancer exhibited a higher B-Raf proto-oncogene serine/threonine kinase (BRAF) mutation frequency compared with rectal cancer, and this may explain the different responses to BRAF-targeting agents. Salem et al (11) demonstrated that catenin $\beta 1$ (CTNNB1) mutations were significantly increased in distal colon cancer compared with rectal cancer, and a further study revealed that tumors containing CTNNB1-mutations were frequently non-polyploid and showed signs of immediate invasive growth (12). Improved understanding of these mutational events and their role in the evolutionary process of cancer may provide insight into the different clinical behaviors of distal colon and rectal cancer.

The Memorial Sloan Kettering-Integrated Mutation Profiling of Actionable Cancer Targets (MSK-IMPACT) is a 
hybridization capture-based next-generation sequencing (NGS) clinical assay for solid tumor molecular oncology (13). In the present study, using the MSK-IMPACT data from cBioPortal, a systematic comparison of molecular alterations between distal colon and rectal cancer was performed. The results of the present study suggested that the mutation profiles of distal colon and rectal cancer were largely similar, but distinct in specific key genetic events, including APC regulator of WNT signaling pathway (APC) R876*, SMAD4 R361 and BRAF mutations.

The findings of the present study may contribute to an improved understanding of the biology of CRC and provide valuable information for improving management of patients with the disease.

\section{Materials and methods}

Data and tumor samples. Data were downloaded from cBioPortal for Cancer Genomics (cbioportal.org/msk-impact). A total of 12,670 tumors from 11,369 unique patients were submitted for MSK-IMPACT sequencing at the Memorial Sloan Kettering Cancer Center (MSKCC) between January 2014 and May 2016 (14). Blood from the same patients was also obtained to serve as a source of matched normal (germline) DNA expression profile. Among the 1,007 CRC samples, 518 were primary tumor samples, although four of these had no clearly annotated tumor origins. Proximal, transverse and rectosigmoid colon cancer were excluded, and 137 distal colon and 125 rectal tumor samples were retained for further analysis.

MSK-IMPACT sequencing workflow. MSK-IMPACT is a comprehensive molecular profiling assay that involves hybridization capture and deep sequencing of all genes that are druggable by approved therapies or are targets of experimental therapies being investigated in clinical trials at MSKCC, as well as frequently mutated genes in human cancer (somatic and germline mutations) (13). Two different panels containing 341 (version 1) and 410 genes (version 2) were used, and all genes from the former panel were included in the latter expanded panel (14). DNA was extracted from tumor and matched normal blood samples using the Chemagic STAR DNA Tissue-10 and Chemagic STAR DNA Blood-400 kits (PerkinElmer, Inc.), respectively. Patient-matched blood DNA was used to identify germline variants. Following sequencing, paired reads were analyzed through a custom bioinformatics pipeline, and the germline variants were filtered out. Each somatic variant identified by the pipeline was manually reviewed to prevent false-positive results $(13,14)$. The alterations were described as suggested in the Human Genome Variation Society (www.hgvs.org/mutnomen). All sequencing work was performed at the MSKCC and reported in the original study (14).

Somatic mutation analysis. Mutation density across the tumors was expressed as number of genetic alterations found in cancer genes present in the MSK-IMPACT panel. Tumor mutational burden (TMB) was calculated as the total number of non-synonymous mutations per megabase $(\mathrm{Mb})$ of the coding region target territory of the assay $(0.98 \mathrm{Mb}$ for version 1 and $1.12 \mathrm{Mb}$ for version 2), and further categorized as low (0-10) or high ( $\geq 10)$. Following the bioinformatics filtering, somatic point mutations were classified as missense, truncating or in-frame mutations according to the predicted protein sequence. Somatic gene mutation rates in distal colon and rectal cancers were calculated, and a frequency $>5 \%$ was considered as significant. The frequencies and hotspot density of specific driver mutations between distal colon and rectal cancer were compared. Mutation plots were generated through adaptation of cBioPortal visualization plots.

Statistical analysis. Statistical analyses were performed using SPSS software (version 22.0; IBM Corp.). Continuous data were described as either the mean \pm standard deviation or median \pm interquartile range (IQR), and categorical variables as counts and frequencies. To compare the differences in patient characteristics and the distribution of gene mutations, Fisher's exact test, $\chi^{2}$ test, paired t-test, or Mann-Whitney U test were used, as appropriate. $\mathrm{P}<0.05$ was considered to indicate a statistically significant difference.

\section{Results}

Tumor characteristics. The mutational profiles of distal colon and rectal cancer were compared using 262 CRC samples, and the clinicopathological features of the patients are summarized in Table I. In the distal colon and rectal cancer groups, $76(53.9 \%)$ and $74(53.0 \%)$ patients were male, respectively ( $P>0.05)$. In addition, no significant difference in smoking history was observed between the two groups $(\mathrm{P}>0.05)$. MSK-IMPACT, an NGS platform for targeted sequencing of cancer-related genes, was performed on all the samples. The average depth of sample coverage for the distal colon and rectal tumors were $740 \mathrm{x}$ and $743 \mathrm{x}$, respectively $(\mathrm{P}>0.05)$. Two types of MSK-IMPACT panels were employed for NGS throughout the study, but there was no apparent distribution difference between the groups.

TMB analysis. TMB was calculated for each sample sequenced for 341/410 genes by MSK-IMPACT. Distal colon tumors had a median of 5.9 mutations/Mb (IQR, 3.0), which was similar to that in the rectal tumors (median $\pm \mathrm{IQR}, 5.9 \pm 4.5$; $\mathrm{P}>0.05)$. It is worth noting that seven cases (5.1\%) in the distal colon group and eight cases $(6.4 \%)$ in the rectal group were tumors with defects in mismatch repair (dMMR) genes (mutL homolog 1, mutS homolog 2, mutS homolog 6 and PMS1 homolog 2 mismatch repair system component), which showed a disproportionately higher number of mutations (55.1 and 52.9 mutations/Mb, respectively). When TMB was calculated for proficient MMR (pMMR) tumors only, the median TMB was 5.9 mutations/ $\mathrm{Mb}$ in both distal colon and rectal tumors, with no significant difference (Mann-Whitney U test, both $\mathrm{P}>0.05$; Fig. 1). The association between TMB and the clinicopathological features of CRC were examined. TMB showed no significant association with sex, smoking history, panel type or sample coverage (all P $>0.05$; Table II). Additionally, the associations remained insignificant after removing dMMR tumors (all P>0.05; Table II).

Driver mutation analysis. Mutational analysis showed that 29 and 21 genes were significantly mutated in distal colon 
Table I. Clinicopathological features of the 262 patients the colorectal tumor samples were obtained from.

\begin{tabular}{lcc}
\hline Clinicopathological feature & Distal colon cancer $(\mathrm{n}=137)$ & Rectal cancer $(\mathrm{n}=125)$ \\
\hline Sex & & $74(53.0 \%)$ \\
Male & $61(53.9 \%)$ & $51(47.0 \%)$ \\
Female & $61(46.1 \%)$ & 0.617 \\
Smoking history & & $54(41.3 \%)$ \\
Previous/Current & $48(37.3 \%)$ & $53(50.9 \%)$ \\
Never & $72(51.3 \%)$ & $18(7.8 \%)$ \\
Unknown & $17(11.4 \%)$ & $24(18.6 \%)$ \\
MSK-IMPACT panel & & $101(81.4 \%)$ \\
IM3_341 genes & $40(30.5 \%)$ & 0.259 \\
IM5_410 genes & $97(76.6 \%)$ & $743 \pm 228$ \\
Sample coverage $(x)$ & & 0.063 \\
Mean \pm SD & $740 \pm 236$ & 0.529 \\
\hline
\end{tabular}

MSK-IMPACT, Memorial Sloan Kettering-Integrated Mutation Profiling of Actionable Cancer Targets.

and rectal tumors, respectively ( $>5 \%$ of tumor samples). Among these genes, 13 significantly mutated genes (SMGs) were shared between the two groups (Fig. 2), including APC, tumor protein p53 (TP53), KRAS proto-oncogene GTPase (KRAS), phosphatidylinositol-4,5-bisphosphate 3-kinase catalytic subunit $\alpha$ (PIK3CA) and SMAD4. Comparison of SMGs between the groups revealed that BRAF mutations were significantly enriched in distal colon cancer (13.9 vs. $4.0 \%$; $\mathrm{P}=0.009$; Fig. 3), whilst SMAD4 mutations were significantly more common in rectal cancer (19.2 vs. $8.8 \%$; $\mathrm{P}=0.019$; Fig. 3). Despite there being no significant difference in the frequencies of KRAS or NRAS proto-oncogene GTPase mutations between the two groups (both P>0.05; Fig. 3), RAS was significantly more frequently mutated in rectal cancer compared with distal colon cancer ( 52.0 vs. $38.0 \%$; $\mathrm{P}=0.025$; Table III). In addition, the data showed that KRAS and BRAF mutations were predominantly, but not completely, exclusive, with only three cases of distal colon and one case of rectal tumor samples carrying both mutations concomitantly. The mutational landscape in the subgroup of pMMR tumors was further examined, and it was demonstrated that these differences in mutational frequencies of BRAF, SMAD4 and RAS between distal colon and rectal tumors remained significant (all $\mathrm{P}<0.05$; Table III).

Mutation hotspot analysis. Mutation hotspot analysis of several key driver genes was performed and it was demonstrated that in both distal colon and rectal cancer, missense mutations were the most common type of point mutations in TP53, KRAS, BRAF, PIK3CA and SMAD4 genes, while truncations were the predominant type of mutations in the APC gene. APC mutations were the most frequent genetic alterations in CRC, and codons 1,286-1,513 (mutation cluster region) were the most commonly mutated loci, covering $~ 40 \%$ of APC mutations in both groups. Additionally, APC R876* was a significant mutation hotspot in rectal cancer compared with distal colon cancer (seen in eight rectal and no distal colon tumor samples; $\mathrm{P}=0.002$; Fig. 4). TP53 mutations were found scattered throughout the coding sequence, but $\sim 25 \%$ of the mutations were clustered at codons R175, R248 and R273 in both groups. For KRAS, G12 and G13 were the predominant hotspots, accounting for 84 and $78 \%$ of KRAS mutations in distal colon and rectal tumors, respectively. For BRAF, $>50 \%$ of the mutations were found clustered at codon V600 in both groups. In PIK3CA, 52 and $35 \%$ of its mutations in distal colon and rectal tumors, respectively, were located at codons R542, R545 and H1047. In addition, similar to APC R876*, SMAD4 R361 missense mutations appeared to be present exclusively in rectal cancer (seen in five rectal and no distal colon tumor samples, respectively; $\mathrm{P}=0.024$; Fig. 5).

\section{Discussion}

Various studies have indicated that CRC is a complex disease with multiple genetic alterations and variable clinical outcomes $(9,15)$. Molecular genotyping of patients with CRC is of vital importance in clinical decision-making regarding diagnostic and therapeutic interventions. In the present study, by comparing the mutational profiles of distal colon and rectal cancer in 262 tumor samples, it was demonstrated that the genetic differences between the two types of cancer were clinically relevant, which emphasized the importance of the location of the primary tumor in the management of $\mathrm{CRC}$ and the implications for future clinical and scientific research.

In the present study, analysis was performed using MSK-IMPACT data with high depth of coverage for improving the understanding of the mutational landscape of distal colon and rectal cancer. TMB analysis showed that the two anatomical locations exhibited similar mutational burdens, and a high-TMB status was present in $14.6 \%$ of distal colon cancer cases and $19.2 \%$ of rectal cancer cases, with no significant difference. MMR-mutated tumors showed a hypermutator phenotype and were most likely to benefit from immune checkpoint blockade therapy (16). After removal of dMMR tumors from analyses, the TMB level in the distal colon and rectal groups remained similar. 


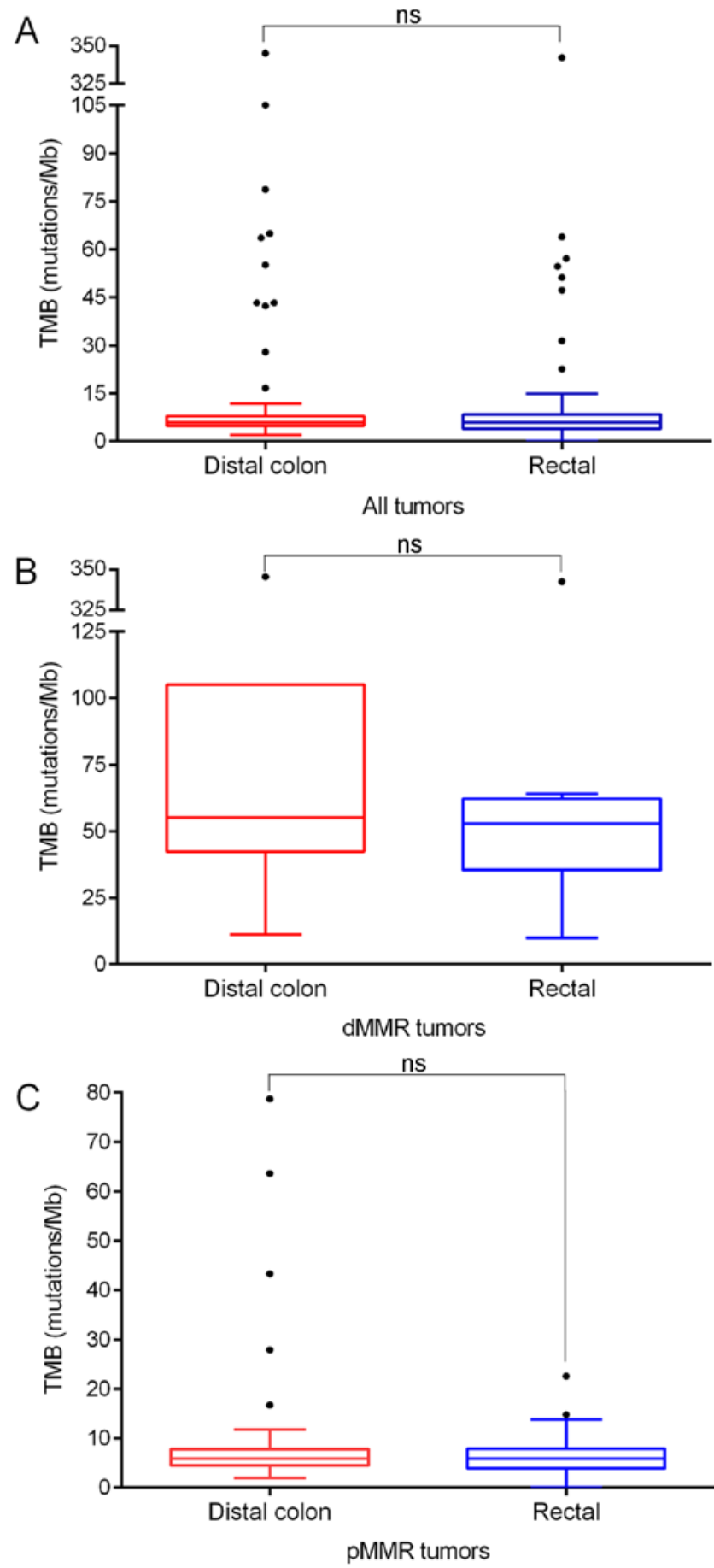

Figure 1. Box plots of TMB in distal colon and rectal cancer according to MMR status in (A) all tumors, (B) dMMR tumors and (C) in pMMR tumors. TMB, tumor mutational burden; MMR, mismatch repair; d, defects; $p$, proficient; $\mathrm{mB}$, mega base.

This finding was in agreement with the result of a previous study (11). In addition, the association of TMB with CRC clinicopathological characteristics was examined, including smoking history, which was reported to be significantly associated with a higher TMB level in lung cancer (17), but no similar association was identified in all the CRC cases in the present study. Previous studies have also suggested that smoking was an independent risk factor for the development of MSI-high CRC $(18,19)$. Therefore, further studies are required to validate the results obtained.
CRC arises through a series of well-characterized histopathological changes as the result of specific genetic 'hits' at certain oncogenes and tumor suppressor genes $(8,20)$. The present study suggested that despite sharing the same critical genomic events, including APC, TP53, KRAS, PIK3CA and SMAD4, there were differences in the frequencies, hotspots and significance of these SMGs in the development of distal colon and rectal cancer. APC and TP53 mutations are the most common genetic alterations in both distal colon and rectal cancer and contribute functionally to various stages of tumor progression $(21,22)$. The present study identified a novel, potentially targetable hotspot mutation in APC R876* that was enriched in rectal cancer compared with distal colon cancer. Ficari et al (23) indicated that the truncation mutation at APC codon 876 , which affected the $\beta$-catenin binding domain, was associated with the density of adenomas of a certain mild colorectal pathophenotype. SMAD4 is an essential mediator in the transforming growth factor- $\beta$ signaling pathway (24), and is associated with CRC metastasis, resistance to 5-fluorouracil chemotherapy and poor outcome $(25,26)$. A study by Mehrvarz et al (27) found that SMAD4 mutations were more frequently detected in colon rather than rectal cancer, and may be associated with the response of CRC to anti-epidermal growth factor receptor (EGFR) therapy. However, the present study observed that SMAD4-mutated tumors were more likely to be located in the rectum than in the distal colon. Furthermore, the SMAD4 R361 mutation was found almost exclusively in rectal cancer and not in distal colon cancer, suggesting that it may be involved in the different clinical and biological behaviors associated with the two different types of CRC, and thus may provide a potential diagnostic or therapeutic target for rectal cancer.

Currently, RAS and BRAF mutation testing has been incorporated into routine clinical practice for patients with CRC receiving anti-EGFR therapy. There is also emerging evidence that PIK3CA mutations are associated with resistance to anti-EGFR therapy $(28,29)$. Sartore-Bianchi et al $(30)$ suggested that a combined mutational analysis of the KRAS and PIK3CA/phosphatase and tensin homolog pathways could identify up to $70 \%$ of patients with advanced CRC who were unlikely to respond to anti-EGFR agents. The results of the present study showed that distal colon and rectal cancer had similar KRAS and PIK3CA mutational status, whereas BRAF and RAS mutations were significantly enriched in distal colon and rectal cancer, respectively. Furthermore, these differences remained significant in the subgroup analysis of pMMR tumors. Similar to the findings of the present study, Salem et al (11) observed that there was a significant decrease in the frequency of BRAF mutations when moving from proximal colon to distal colon to the rectum, suggesting that different underlying mechanisms may be involved in rectal cancer and distal colon cancer. In addition, in the present study it was observed that mutations in KRAS and BRAF were primarily, but not completely, mutually exclusive in both distal colon and rectal cancer, thus differing from the majority of previous reports $(31,32)$. However, the exclusivity of the mutational status of KRAS and BRAF may be largely due to the high-depth sequencing coverage of the MSK-IMPACT assay, which can detect mutations that appear only in a minority of cells in a sample (14). 
Table II. Association between TMB and the clinicopathological features of the patients the colorectal tumor samples were obtained from.

\begin{tabular}{|c|c|c|c|c|c|c|}
\hline \multirow{2}{*}{$\begin{array}{l}\text { Clinicopathological } \\
\text { feature }\end{array}$} & \multicolumn{3}{|c|}{ All colorectal tumors } & \multicolumn{3}{|c|}{ pMMR colorectal tumors } \\
\hline & Low TMB $(n=218)$ & High TMB $(n=44)$ & P-value & Low TMB (n=218) & High TMB $(n=29)$ & P-value \\
\hline \multicolumn{7}{|l|}{ Sex } \\
\hline Male & $127(58.3 \%)$ & $23(52.3 \%)$ & & $127(58.3 \%)$ & $12(41.4 \%)$ & \\
\hline Female & $91(41.7 \%)$ & $21(47.7 \%)$ & 0.506 & $91(41.7 \%)$ & $17(58.6 \%)$ & 0.111 \\
\hline \multicolumn{7}{|l|}{ Smoking history } \\
\hline Previous/Current & $87(39.9 \%)$ & $15(34.1 \%)$ & & $87(39.9 \%)$ & $8(27.6 \%)$ & \\
\hline Never & $102(46.8 \%)$ & $23(52.3 \%)$ & & $102(46.8 \%)$ & $16(55.2 \%)$ & \\
\hline Unknown & $29(13.3 \%)$ & $6(13.5 \%)$ & 0.742 & $29(13.3 \%)$ & $5(17.2 \%)$ & 0.412 \\
\hline \multicolumn{7}{|l|}{ MSK-IMPACT panel } \\
\hline IM3_341 genes & $50(22.9 \%)$ & $14(31.8 \%)$ & & $50(22.9 \%)$ & $11(37.9 \%)$ & \\
\hline IM5_410 genes & $168(77.1 \%)$ & $30(68.2 \%)$ & 0.248 & $168(77.1 \%)$ & $18(62.1 \%)$ & 0.106 \\
\hline \multicolumn{7}{|l|}{ Sample coverage $(\mathrm{x})$} \\
\hline Mean \pm SD & $745 \pm 238$ & $700 \pm 218$ & 0.073 & $745 \pm 238$ & $722 \pm 234$ & 0.268 \\
\hline
\end{tabular}

TMB, tumor mutational burden; pMMR, proficient mismatch repair; low TMB, $<10.0$ mutations/Mb; high TMB, $\geq 10.0$ mutations/Mb; MSK-IMPACT, Memorial Sloan Kettering-Integrated Mutation Profiling of Actionable Cancer Targets.

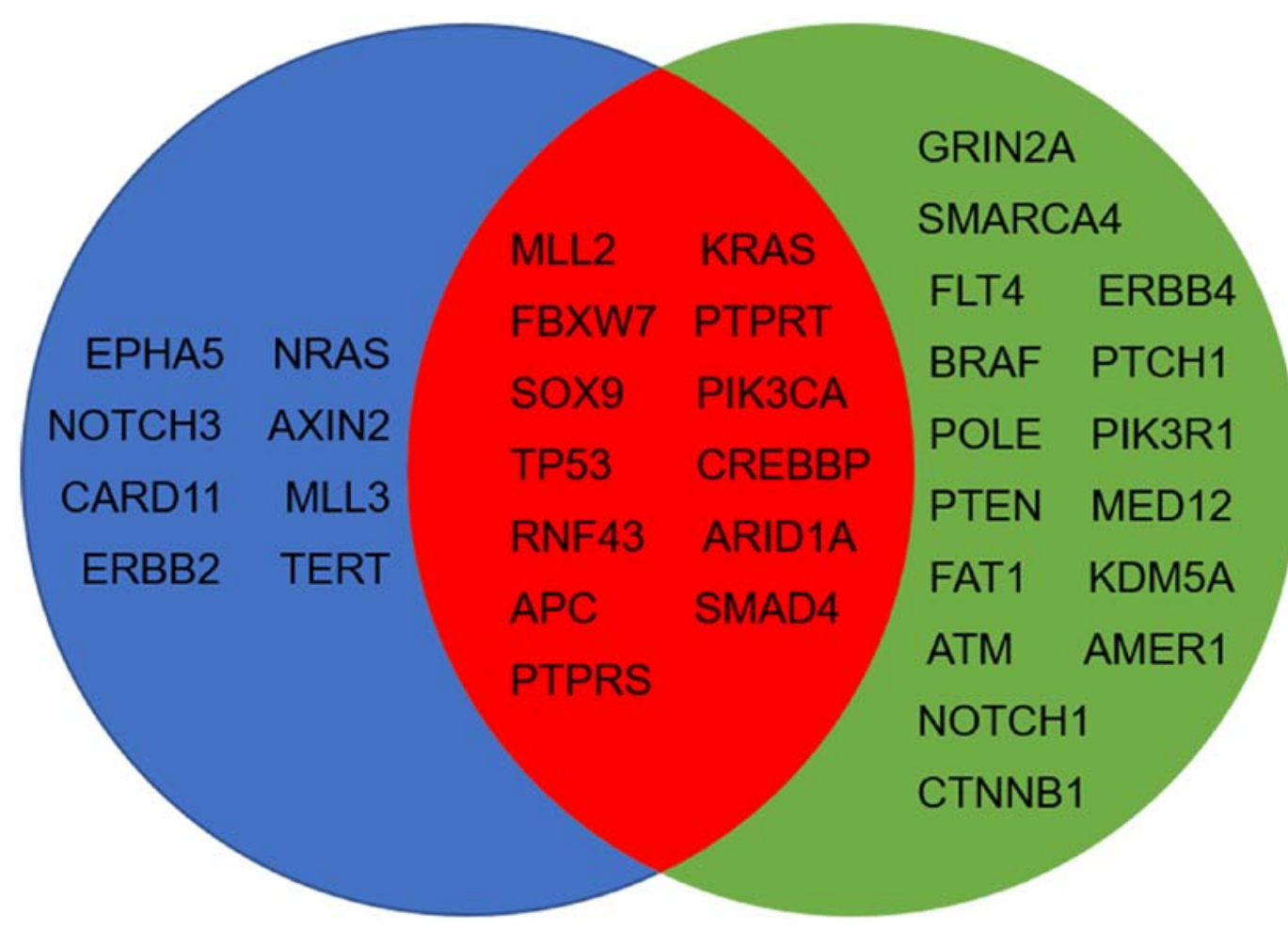

Rectal cancer

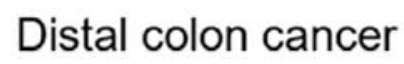

Figure 2. Venn diagram of SMGs in distal colon and rectal cancer. A total of 13 SMGs were shared between distal colon and rectal tumors and were marked red; 16 SMGs were private to distal colon cancer and were marked green; 8 SMGs were private to rectal cancer and were marked blue. Ns, not significant; SMGs, significantly mutated genes.

In conclusion, despite the limitation that the present study was primarily computational and requires further experimental validation, the results suggested that the mutation profiles of distal colon and rectal cancer are similar in principle, but distinct 
Table III. Enrichment analysis of BRAF, SMAD4 and RAS mutations between distal colon and rectal cancer.

\begin{tabular}{|c|c|c|c|c|c|c|}
\hline \multirow[b]{2}{*}{ Gene } & \multicolumn{3}{|c|}{ All colorectal tumors } & \multicolumn{3}{|c|}{ pMMR colorectal tumors } \\
\hline & $\begin{array}{l}\text { Distal colon } \\
(n=137)(\%)\end{array}$ & $\begin{array}{c}\text { Rectal } \\
(n=125)(\%)\end{array}$ & $\mathrm{P}$-value & $\begin{array}{l}\text { Distal colon } \\
(n=130)(\%)\end{array}$ & $\begin{array}{c}\text { Rectal } \\
(n=117)(\%)\end{array}$ & P-value \\
\hline \multicolumn{7}{|l|}{ BRAF } \\
\hline Mutant & $19(13.9)$ & $5(4.0)$ & & $16(12.3)$ & $4(3.4)$ & \\
\hline Wild-type & $118(86.1)$ & $120(96.0)$ & 0.009 & $114(87.7)$ & $113(96.6)$ & 0.018 \\
\hline \multicolumn{7}{|l|}{ SMAD4 } \\
\hline Mutant & $12(8.8)$ & $24(19.2)$ & & $12(9.2)$ & $24(20.5)$ & \\
\hline Wild-type & $125(91.2)$ & $101(80.8)$ & 0.019 & $118(90.8)$ & $93(79.5)$ & 0.018 \\
\hline \multicolumn{7}{|c|}{ RAS (KRAS/NRAS) } \\
\hline Mutant & $52(38.0)$ & $65(52.0)$ & & 49 (37.7) & $60(51.3)$ & \\
\hline Wild-type & $85(62.0)$ & $60(48.0)$ & 0.025 & $81(62.3)$ & $57(48.7)$ & 0.040 \\
\hline
\end{tabular}

BRAF, B-Raf proto-oncogene serine/threonine kinase; SMAD4, SMAD family member 4; KRAS, KRAS proto-oncogene GTPase; NRAS, NRAS proto-oncogene GTPase; pMMR, proficient mismatch repair.

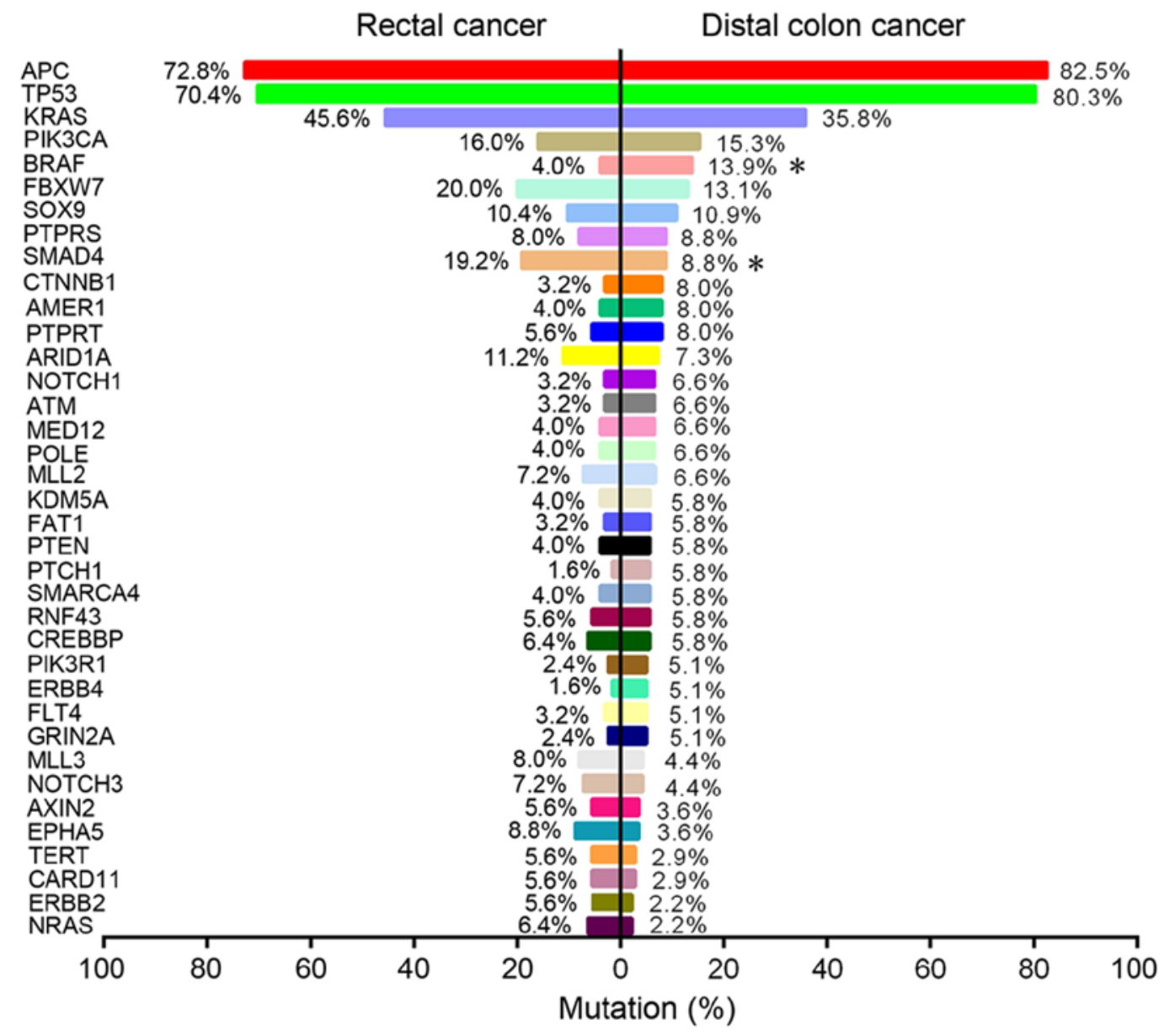

Figure 3. Comparison of significantly mutated genes between distal colon and rectal cancer. * $<0.05$, among the 37 genes, only BRAF and SMAD4 showed significantly different mutational frequencies between distal colon and rectal cancers. BRAF, B-Raf proto-oncogene serine/threonine kinase; SMAD4, SMAD family member 4 .

in specific key genetic events, including APC R876*, SMAD4 R361 and BRAF mutations. Therefore, the findings of the present study may contribute to understanding the differences in tumor biology and clinical behavior between distal colon and rectal cancer. The present study highlighted the necessity to consider distal colon and rectal cancer in the context of genetic 

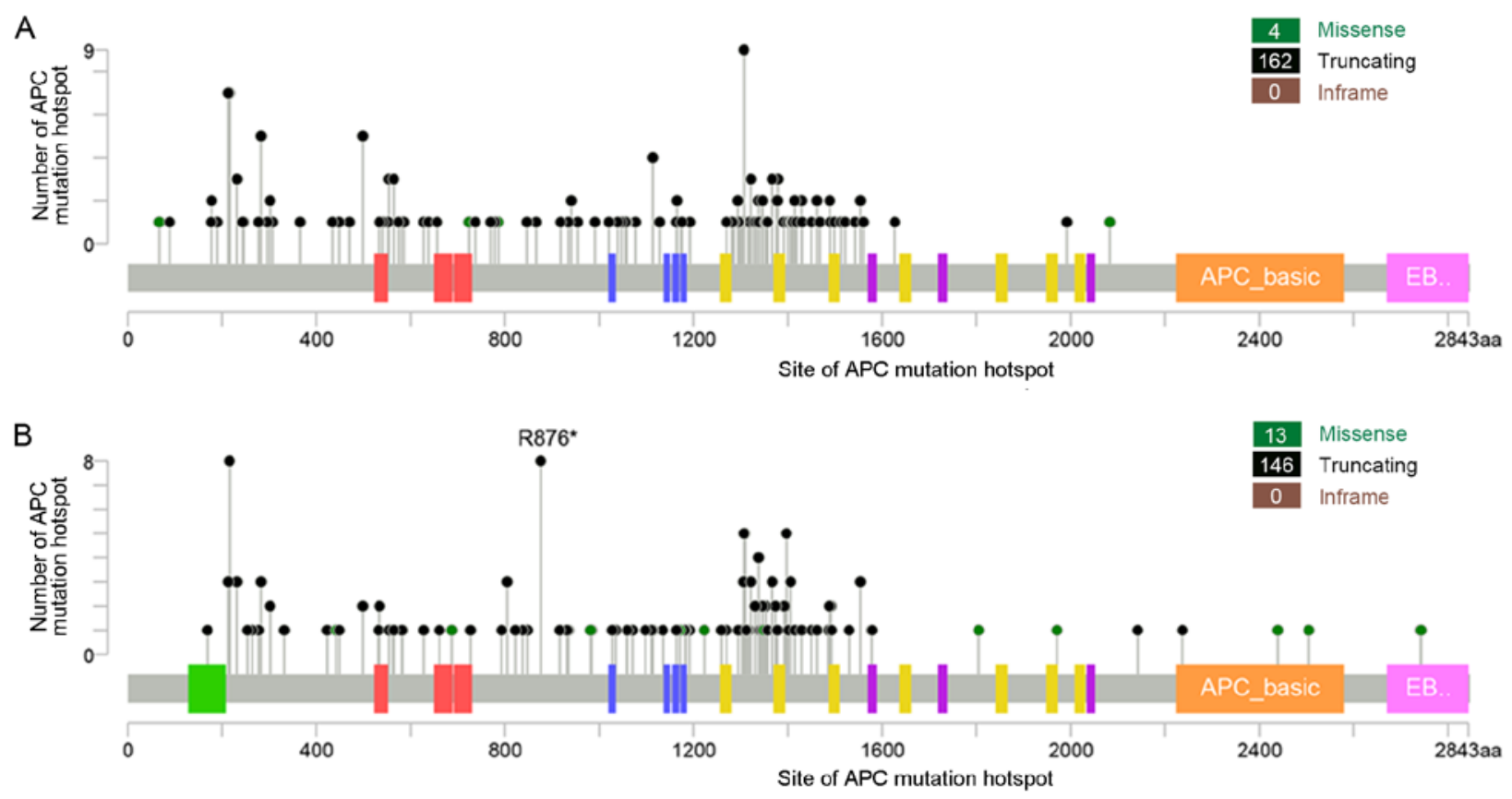

Figure 4. APC gene mutation status in (A) distal colon and (B) rectal cancer. APC, APC regulator of WNT signaling pathway.

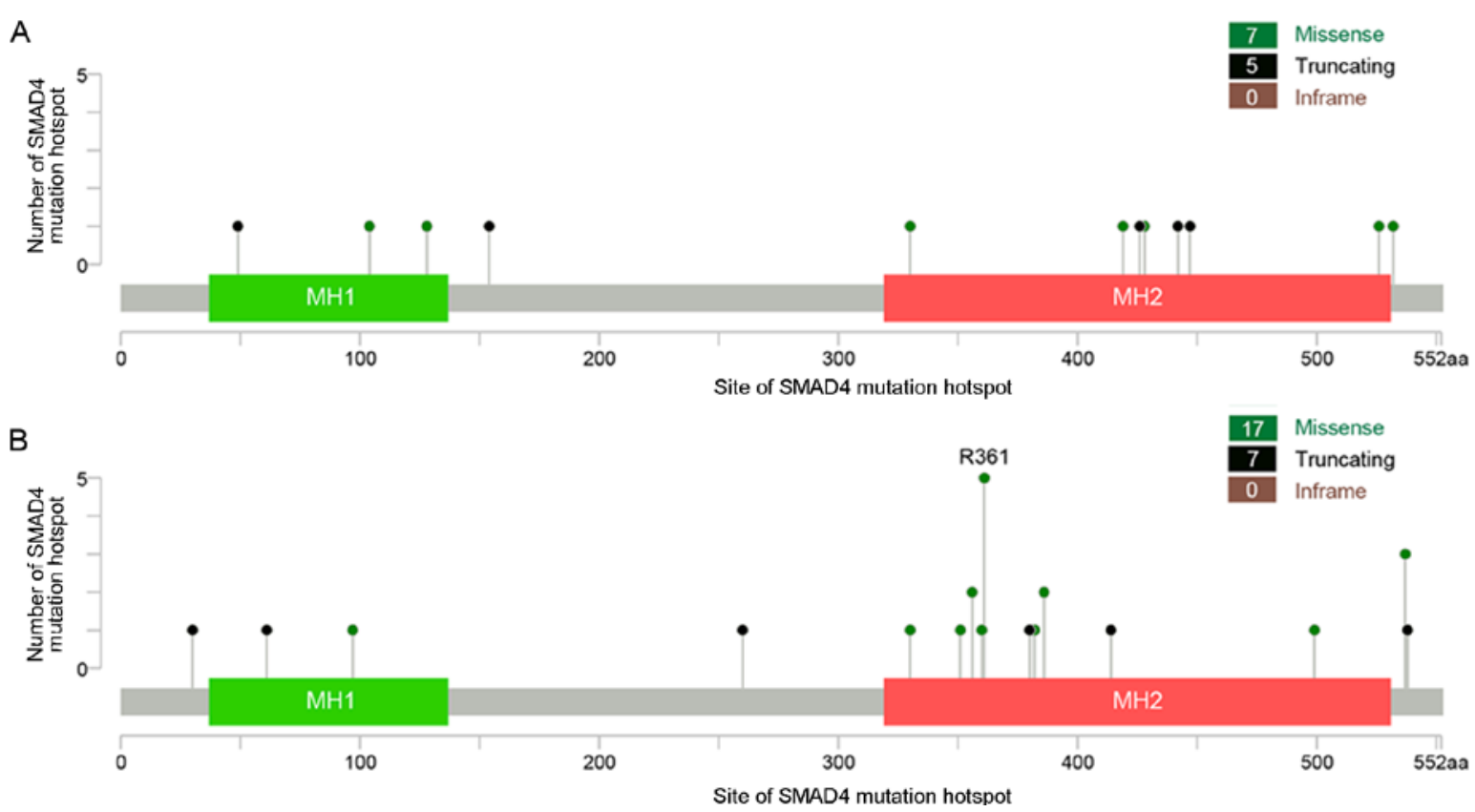

Figure 5. SMAD4 gene mutation status in distal colon (A) and rectal (B) cancer. SMAD4, SMAD family member 4.

background when selecting treatment regimens, designing research trials and analyzing clinical outcomes.

\section{Acknowledgements}

Not applicable.

\section{Funding}

The present study was funded by the National Natural Science Foundation of China (grant no. 81874201) and Shanghai Science and Technology Commission (grant no. 19411971500).

\section{Availability of data and materials}

The datasets generated and/or analyzed during the current study are available from cBioPortal for Cancer Genomics at cbioportal.org/msk-impact.

\section{Authors' contributions}

$\mathrm{ZZ}$ and $\mathrm{HJ}$ designed the study. ZZ, AW and XT performed the research. YC, ET and $\mathrm{HJ}$ contributed to the data analysis. HJ supervised the study. ZZ and AW drafted the manuscript. All authors read and approved the final manuscript. 


\section{Ethics approval and consent to participate}

Not applicable.

\section{Patient consent for publication}

Not applicable.

\section{Competing interests}

The authors declare that they have no competing interests.

\section{References}

1. Torre LA, Bray F, Siegel RL, Ferlay J, Lortet-Tieulent J and Jemal A: Global cancer statistics, 2012. CA Cancer J Clin 65 $87-108,2015$

2. Sugai T, Habano W, Jiao YF, Tsukahara M, Takeda Y, Otsuka K and Nakamura S: Analysis of molecular alterations in left- and right-sided colorectal carcinomas reveals distinct pathways of carcinogenesis: Proposal for new molecular profile of colorectal carcinomas. J Mol Diagn 8: 193-201, 2006.

3. Lee GH, Malietzis G, Askari A, Bernardo D, Al-Hassi HO and Clark SK: Is right-sided colon cancer different to left-sided colorectal cancer? A systematic review. Eur J Surg Oncol 41 300-308, 2015

4. Takahashi Y, Sugai T, Habano W, Ishida K, Eizuka M, Otsuka K, Sasaki A, Takayuki M, Morikawa T, Unno M and Suzuki H: Molecular differences in the microsatellite stable phenotype between left-sided and right-sided colorectal cancer. Int J Cancer 139: 2493-2501, 2016.

5. Minoo P, Zlobec I, Peterson M, Terracciano L and Lugli A Characterization of rectal, proximal and distal colon cancers based on clinicopathological, molecular and protein profiles. Int J Oncol 37: 707-718, 2010.

6. Gao XH, Yu GY, Gong HF, Liu LJ, Xu Y, Hao LQ, Liu P, Liu ZH, Bai CG and Zhang W: Differences of protein expression profiles, KRAS and BRAF mutation, and prognosis in right-sided colon, left-sided colon and rectal cancer. Sci Rep 7: 7882, 2017.

7. Salem ME, Yin J, Weinberg BA, Renfro LA, Pederson LD, Maughan TS, Adams RA, Van Cutsem E, Falcone A, Tebbutt NC, et al: Clinicopathological differences and survival outcomes with first-line therapy in patients with left-sided colon cancer and rectal cancer: Pooled analysis of 2,879 patients from AGITG (MAX), COIN, FOCUS2, OPUS, CRYSTAL and COIN-B trials in the ARCAD database. Eur J Cancer 103: 205-213, 2018

8. Bogaert J and Prenen H: Molecular genetics of colorectal cancer. Ann Gastroenterol 27: 9-14, 2014.

9. Worthley DL and Leggett BA: Colorectal cancer: Molecular features and clinical opportunities. Clin Biochem Rev 31: 31-38, 2010.

10. Tie J, Gibbs P, Lipton L, Christie M, Jorissen RN, Burgess AW, Croxford M, Jones I, Langland R, Kosmider S, et al: Optimizing targeted therapeutic development: Analysis of a colorectal cancer patient population with the BRAF(V600E) mutation. Int J Cancer 128: 2075-2084, 2011.

11. Salem ME, Weinberg BA, Xiu J, El-Deiry WS, Hwang JJ, Gatalica Z, Philip PA, Shields AF, Lenz HJ and Marshall JL: Comparative molecular analyses of left-sided colon, right-sided colon, and rectal cancers. Oncotarget 8: 86356-86368, 2017.

12. Ahadova A, von Knebel Doeberitz M, Bläker H and Kloor M: CTNNB1-mutant colorectal carcinomas with immediate invasive growth: A model of interval cancers in Lynch syndrome. Fam Cancer 15: 579-586, 2016.

13. Cheng DT, Mitchell TN, Zehir A, Shah RH, Benayed R, Syed A Chandramohan R, Liu ZY, Won HH, Scott SN, et al: Memorial Sloan Kettering-integrated mutation profiling of actionable cancer targets (MSK-IMPACT): A hybridization capture-based next-generation sequencing clinical assay for solid tumor molecular oncology. J Mol Diagn 17: 251-264, 2015.

14. Zehir A, Benayed R, Shah RH, Syed A, Middha S, Kim HR, Srinivasan P, Gao J, Chakravarty D, Devlin SM, et al: Mutational landscape of metastatic cancer revealed from prospective clinical sequencing of 10,000 patients. Nat Med 23: 703-713, 2017.
15. Phipps AI, Limburg PJ, Baron JA, Burnett-Hartman AN, Weisenberger DJ, Laird PW, Sinicrope FA, Rosty C, Buchanan DD, Potter JD and Newcomb PA: Association between molecular subtypes of colorectal cancer and patient survival. Gastroenterology 148: 77-87.e2, 2015.

16. Le DT, Uram JN, Wang H, Bartlett BR, Kemberling $\mathrm{H}$, Eyring AD, Skora AD, Luber BS, Azad NS, Laheru D, et al: PD-1 blockade in tumors with mismatch-repair deficiency. N Engl J Med 372: 2509-2520, 2015

17. Govindan R, Ding L, Griffith M, Subramanian J, Dees ND, Kanchi KL, Maher CA, Fulton R, Fulton L, Wallis J, et al: Genomic landscape of non-small cell lung cancer in smokers and never-smokers. Cell 150: 1121-1134, 2012.

18. Slattery ML, Curtin K, Anderson K, Ma KN, Ballard L, Edwards S, Schaffer D, Potter J, Leppert $M$ and Samowitz WS: Associations between cigarette smoking, lifestyle factors, and microsatellite instability in colon tumors. J Natl Cancer Inst 92: 1831-1836, 2000.

19. Chia VM, Newcomb PA, Bigler J, Morimoto LM, Thibodeau SN and Potter JD: Risk of microsatellite-unstable colorectal cancer is associated jointly with smoking and nonsteroidal anti-inflammatory drug use. Cancer Res 66: 6877-6883, 2006.

20. Wood LD, Parsons DW, Jones S, Lin J, Sjöblom T, Leary RJ, Shen D, Boca SM, Barber T, Ptak J, et al: The genomic landscapes of human breast and colorectal cancers. Science 318: 1108-1113, 2007.

21. Fodde R: The APC gene in colorectal cancer. Eur J Cancer 38: 867-871, 2002

22. Iacopetta B: TP53 mutation in colorectal cancer. Hum Mutat 21: 271-276, 2003.

23. Ficari F, Cama A, Valanzano R, Curia MC, Palmirotta R, Aceto G, Esposito DL, Crognale S, Lombardi A, Messerini L, et al: APC gene mutations and colorectal adenomatosis in familial adenomatous polyposis. Br J Cancer 82: 348-353, 2000.

24. Xu J and Attisano L: Mutations in the tumor suppressors Smad2 and Smad4 inactivate transforming growth factor beta signaling by targeting Smads to the ubiquitin-proteasome pathway. Proc Natl Acad Sci USA 97: 4820-4825, 2000.

25. Li X, Liu B, Xiao J, Yuan Y, Ma J and Zhang Y: Roles of VEGF-C and Smad4 in the lymphangiogenesis, lymphatic metastasis, and prognosis in colon cancer. J Gastrointest Surg 15: 2001-2010, 2011.

26. Zhang B, Zhang B, Chen X, Bae S, Singh K, Washington MK and Datta PK: Loss of Smad4 in colorectal cancer induces resistance to 5-fluorouracil through activating Akt pathway. Br J Cancer 110: 946-957, 2014.

27. Mehrvarz Sarshekeh A, Advani S, Overman MJ, Manyam G, Kee BK, Fogelman DR, Dasari A, Raghav K, Vilar E, Manuel S, et al: Association of SMAD4 mutation with patient demographics, tumor characteristics, and clinical outcomes in colorectal cancer. PLoS One 12: e0173345, 2017.

28. Therkildsen C, Bergmann TK, Henrichsen-Schnack T, Ladelund $S$ and Nilbert $M$ : The predictive value of KRAS, NRAS, BRAF, PIK3CA and PTEN for anti-EGFR treatment in metastatic colorectal cancer: A systematic review and meta-analysis. Acta Oncol 53: 852-864, 2014

29. Mao C, Yang ZY, Hu XF, Chen Q and Tang JL: PIK3CA exon 20 mutations as a potential biomarker for resistance to anti-EGFR monoclonal antibodies in KRAS wild-type metastatic colorectal cancer: A systematic review and meta-analysis. Ann Oncol 23: $1518-1525,2012$

30. Sartore-Bianchi A, Martini M, Molinari F, Veronese S, Nichelatti M, Artale S, Di Nicolantonio F, Saletti P, De Dosso S, Mazzucchelli L, et al: PIK3CA mutations in colorectal cancer are associated with clinical resistance to EGFR-targeted monoclonal antibodies. Cancer Res 69: 1851-1857, 2009.

31. De Roock W, Claes B, Bernasconi D, De Schutter J, Biesmans B, Fountzilas G, Kalogeras KT, Kotoula V, Papamichael D, Laurent-Puig P, et al: Effects of KRAS, BRAF, NRAS, and PIK3CA mutations on the efficacy of cetuximab plus chemotherapy in chemotherapy-refractory metastatic colorectal cancer: A retrospective consortium analysis. Lancet Oncol 11: 753-762, 2010.

32. Italiano A, Hostein I, Soubeyran I, Fabas T, Benchimol D, Evrard S, Gugenheim J, Becouarn Y, Brunet R, Fonck M, et al: KRAS and BRAF mutational status in primary colorectal tumors and related metastatic sites: Biological and clinical implications. Ann Surg Oncol 17: 1429-1434, 2010.

This work is licensed under a Creative Commons Attribution-NonCommercial-NoDerivatives 4.0 International (CC BY-NC-ND 4.0) License. 